\title{
Análise econômica do processo de recuperação de um solo sódico no Perímetro Irrigado Curu-Pentecoste, CE
}

\author{
Ana P. B. de Araújo ${ }^{1}$, Raimundo N. T. Costa ${ }^{1}$, Claudivan F. de Lacerda ${ }^{1} \&$ Hans R. G heyi ${ }^{2}$
}

\section{RESUMO}

A pesquisa teve como objetivo analisar, com base nos indicadores de rentabilidade da análise de investimento, a viabilidade econômica do processo de recuperação de um solo sódico no Perímetro Irrigado Curu-Pentecoste-CE. O delineamento experimental foi em blocos inteiramente casualizados com cinco tratamentos e cinco repetições. 0 s tratamentos de recuperação foram: $T_{0}$ : testemunha; $T_{1}: 40$ tha ${ }^{-1}$ de matéria orgânica (M .O ); $T_{2} 20$ tha $^{-1}$ de gesso; $T_{3}: 20$ t ha ${ }^{-1}$ gesso +40 t ha ${ }^{-1}$ M.O .; $T_{4}: 10$ t ha- ${ }^{-1}$ gesso + 20 t ha $^{-1}$ M.O. A análise dos indicadores de rentabilidade foi realizada de forma "ex-ante" e "ex-post", considerando-se os custos e/ou investimentos na recuperação do solo sódico e instalação futura de um sistema de drenagem subterrânea. 0 cultivo do feijão-de-corda contribuiu com apenas $12 \%$ do processo de recuperação do solo sódico. Do ponto de vista estritamente financeiro e na alternativa da aplicação de um único melhorador, o gesso se apresentou como a alternativa mais atrativa. o processo de recuperação apresentou viabilidade econômica a uma taxa de juros de $12 \%$ ao ano, porém, considerandose que o "payback" foi de nove anos, há a necessidade da criação de uma linha de crédito subsidiada, destinada a agricultores familiares como forma de incorporar, ao processo produtivo, as extensas áreas de solos degradados por sódio.

Palavras-chave: salinidade, feijão-de-corda, relação custo benefício

\section{Economical analysis of the reclamation of a sodic soil in the Irrigated Perimeter Curu-Pentecoste, CE}

\section{ABSTRACT}

The objective of the present study was to evaluate on the basis of profitability indicators, the economic viability of the reclamation processes of a sodic soil located at the Irrigated Perimeter Curu-Pentecoste, $\mathrm{CE}$, relating the reclamation cost with the gross revenue obtained from the cowpea. The experimental design was a completely randomized block with five treatments and five replications. The reclamation treatments were $T_{0}$ : control, $T_{1}: 40$ t ha $^{-1}$ of organic matter $(O M), T_{2}: 20$ t gypsum ha ${ }^{-1}, T_{3}: 20$ t ha $a^{-1}$ gypsum $+^{-}$ $40 \mathrm{t} \mathrm{ha}^{-1} \mathrm{MO}, \mathrm{T}_{4}: 10 \mathrm{t} \mathrm{ha}^{-1}$ gypsum $+20 \mathrm{t} \mathrm{ha}^{-1} \mathrm{MO}$. The profitability indicators were analysed through an "ex-ante" and an "ex-post" evaluation forms, considering the costs and/or investments on the sodic soil reclamation and the installation of an underground drainage system. The cowpea yield contributed only with $12 \%$ of the reclamation costs of the sodic soil. From a strictly financial point of view, and considering the alternative of applying only one soil amendment, the gypsum appeared as the most attractive al ternative. The reclamation process showed a good economic viability with an interest rate of $12 \%$ per year and high economic viability with a rate of $6 \%$ per year, demonstrating the necessity of a creation of a policy of subsidized credit as a way to incorporate the extensive areas of degraded sodic soil to productive process.

Key words: salinity, cowpea, cost benefit ratio 


\section{INTRODUÇÃO}

O feijão caupi (Vigna unguiculata (L.) Walp.) é uma espécie bem adaptada às condições do semiárido, onde frequentemente prevalecem condições adversas de seca, salinidade, temperaturas elevadas e alta insolação. É possuidora de uma ampla variabilidade genética, ampla capacidade de adaptação, alto potencial produtivo e excelente valor nutritivo (Freire Filho et al., 2005).

Extensas áreas irrigadas em todo o mundo vêm apresentando diminuição de suas produções em decorrência do excesso de sais (Keiffer \& Ungar, 2002; Horney et al., 2005; Leal et al., 2008). No Brasil, em especial no Nordeste, aproximadamente 25\% das áreas irrigadas já foram salinizados (Gheyi, 2000).

O problema da salinidade nas regiões semiáridas tem-se agravado e tende a se tornar um problema de difícil solução em virtude de fatores inerentes ao clima, à geologia, à qualidade da água e ao manejo da irrigação. O excesso de sais solúveis leva à redução do potencial osmótico da solução do solo, causando dificuldades no processo de absorção de água pela planta e desbalanço nutricional, afetando o desenvolvimento das culturas (Dutra et al., 2000; Viana et al., 2001; Amorim et al., 2002).

Segundo Costa et al. (2005), a recuperação de solos afetados por sais deve seguir uma aplicação criteriosa de tecnologia específica desenvolvida para as condições peculiares de cada região, visando propiciar condições favoráveis de umidade, aeração e balanço de sais ao sistema radicular das culturas. Faz-se necessário, portanto, a instalação de um sistema de drenagem subterrânea em áreas irrigadas, o que constitui uma das principais infraestruturas no processo de dessalinização dos solos, além de se fazer uso de corretivos que contenham cálcio solúvel, bem como práticas mecânicas adequadas.

Melo et al. (2008), relatam que a salinização e/ou sodificação do solo é responsável pela redução na produção agrícola, culminando quase sempre com o abandono de áreas agricultáveis, acarretando grandes prejuízos à economia regional. Conforme o autor, a recuperação desses solos é imprescindível para que eles sejam reincorporados ao sistema produtivo.

A recuperação de solos com problemas de salinidade e/ou sodicidade, pode ser realizada através de técnicas de natureza física ou mecânica, química e biológica. As técnicas mecânicas utilizam as práticas de subsolagem, aração e gradagem. As químicas usam corretivos como o gesso, o cloreto de cálcio, o enxofre e o ácido sulfúrico. E as biológicas utilizam plantas e/ ou resíduo orgânico que auxiliam nas propriedades físicas do solo (Batista et al., 2002).

Sales et al. (2004) constataram que a introdução de um sistema de drenagem subterrânea proporcionou uma redução nos níveis iniciais de salinidade do solo para uma faixa que não representava risco de redução na produtividade da cultura da videira.

Para uma análise econômica é conveniente fazer estimativas de todas as entradas e saídas, ou seja; todos os custos envolvidos no investimento inicial, operação e manutenção, tal como as receitas geradas durante determinado período de tempo. Obtém-se, deste modo, o fluxo de caixa financeiro relativo à atividade, permitindo o cálculo dos indicadores econômicos obtidos com o empreendimento. Alguns dos principais indicadores são: a relação benefício/custo (B/C) a TIR - Taxa interna de retorno; o TRC- Tempo de retorno de capital e o VPL - Valor presente líquido (Sartori, 2007).

A pesquisa teve como objetivo analisar, com base nos indicadores de rentabilidade da análise de investimento, a viabilidade econômica do processo de recuperação de um solo sódico no Perímetro Irrigado Curu-Pentecoste-CE.

\section{Material e MÉTOdos}

A pesquisa foi realizada no período de julho de 2007 a janeiro de 2009, no Núcleo D do Perímetro Irrigado Curu Pentecoste, $\mathrm{CE}$, em área de 105 x $105 \mathrm{~m}$. O solo apresenta textura francoargilosa, classificado como Neossolo flúvico com percentagem de sódio trocável em torno de $50 \%$, porém sem problemas de sais solúveis (Tabela 1). O clima da região, de acordo com a classificação de Köeppen, é do tipo BSw'h', quente e semiárido com chuvas irregulares distribuídas de fevereiro a maio, com precipitação pluvial medial anual de $801 \mathrm{~mm}$, evaporação de $1475 \mathrm{~mm}$; temperatura média anual em torno de $27,1^{\circ} \mathrm{C}$ e umidade relativa média do ar de $73,7 \%$.

O preparo de solo da área do experimento constou, inicialmente, de uma aração seguida de uma gradagem, com vistas a um ajuste no plano sistematizado do terreno para fins de melhorar as condições de escoamento nos sulcos de irrigação.

Tabela 1. Atributos físicos e químicos do solo da área do experimento

\begin{tabular}{|c|c|c|c|c|c|c|c|c|c|}
\hline \multicolumn{5}{|c|}{ Composição granulométrica $\left(\mathrm{g} \mathrm{kg}^{-1}\right)$} & \multirow{2}{*}{$\begin{array}{l}\text { Classe } \\
\text { textural }\end{array}$} & \multirow{2}{*}{$\begin{array}{c}\text { Densidade } \\
\text { do solo }\left(\mathrm{kg} \mathrm{m}^{-3}\right)\end{array}$} & \multirow{2}{*}{ pH } & \multirow{2}{*}{\multicolumn{2}{|c|}{$\begin{array}{c}\text { CE } \\
\left(d S \mathrm{~m}^{-1}\right)\end{array}$}} \\
\hline Prof. (cm) & Areia grossa & Areia fina & Silte & Argila & & & & & \\
\hline $\begin{array}{c}0-30 \\
30-60 \\
60-90\end{array}$ & $\begin{array}{l}50 \\
50 \\
50\end{array}$ & $\begin{array}{l}250 \\
250 \\
450\end{array}$ & $\begin{array}{l}470 \\
390 \\
300\end{array}$ & $\begin{array}{l}230 \\
310 \\
200\end{array}$ & $\begin{array}{c}\text { Franca } \\
\text { Franco- argilosa } \\
\text { Franca }\end{array}$ & $\begin{array}{l}1430 \\
1540 \\
1500\end{array}$ & $\begin{array}{l}9,4 \\
9,2 \\
9,5\end{array}$ & & \\
\hline $\mathrm{Ca}^{2+}$ & $\mathrm{Mg}^{2+}$ & & & $\begin{array}{c}\text { Complex } \\
\mathrm{Na}^{+}\end{array}$ & $\begin{array}{r}0 \text { Sortivo } \text { (cmol }_{\mathrm{c}} \mathbf{~ k g} \\
\mathrm{H}^{+}+\mathrm{Al}^{3+}\end{array}$ & $\mathbf{A} \mathbf{l}^{3+}$ & & S & $T$ \\
\hline $\begin{array}{c}8,00 \\
7,60 \\
10,00 \\
\end{array}$ & $\begin{array}{l}3,00 \\
2,60 \\
2,00 \\
\end{array}$ & & & $\begin{array}{l}13,62 \\
14,86 \\
10,73 \\
\end{array}$ & $\begin{array}{l}0,00 \\
0,00 \\
0,00 \\
\end{array}$ & $\begin{array}{l}0,00 \\
0,00 \\
0,00 \\
\end{array}$ & & $\begin{array}{l}25,0 \\
25,4 \\
22,9 \\
\end{array}$ & $\begin{array}{l}25,0 \\
25,4 \\
22,9 \\
\end{array}$ \\
\hline $\mathrm{V}(\%)$ & PST & $\mathrm{C}(\mathrm{c}$ & & $\mathrm{N}\left(\mathrm{g} \mathrm{kg}^{-1}\right)$ & $\mathrm{C} / \mathrm{N}$ & M $0\left(\mathrm{~g} \mathrm{~kg}^{-1}\right)$ & & P As & $\left.\lg \mathbf{k g}^{-1}\right)$ \\
\hline $\begin{array}{l}100 \\
100 \\
100\end{array}$ & $\begin{array}{l}54 \\
58 \\
47\end{array}$ & & & $\begin{array}{l}0,51 \\
0,61 \\
0,41\end{array}$ & $\begin{array}{l}10 \\
10 \\
10\end{array}$ & $\begin{array}{c}8,68 \\
10,34 \\
6,93\end{array}$ & & & \\
\hline
\end{tabular}


Considerando as elevadas percentagens de sódio trocável e, portanto, a presença de camadas retardadoras do fluxo de água, traduzidas nos valores de condutividade hidráulica do solo saturado praticamente nulo, toda a área do experimento foi subsolada até uma profundidade de 0,60 m (Figura 1); posteriormente, realizaram-se uma nova gradagem e o sulcamento, tendo em vista a irrigação ser realizada pelo sistema de sulcos.

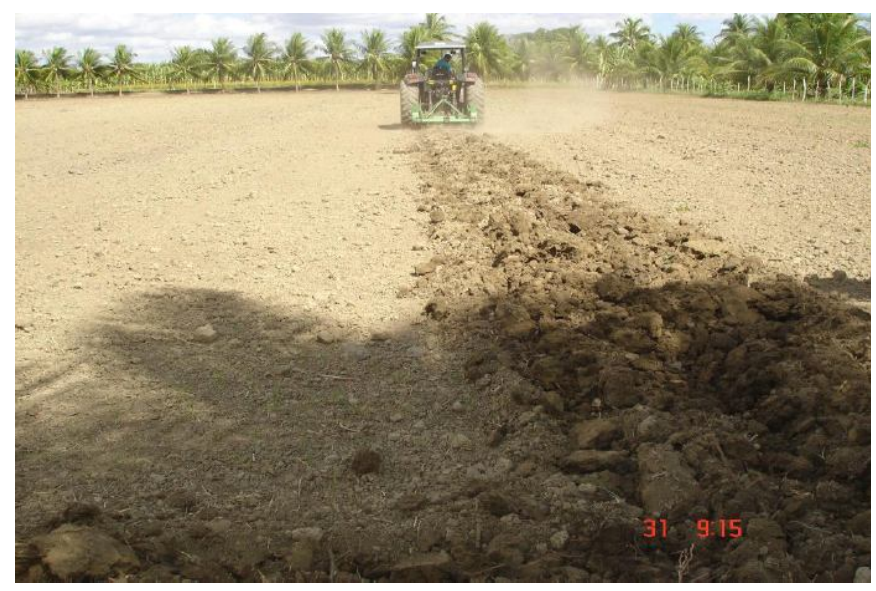

Figura 1. Subsolagem da área do experimento

A irrigação através do sistema de sulcos era realizada com frequência semanal, utilizando-se, como critério do tempo de reposição, a metade do tempo de avanço da água no sulco. Uma área de 3,0 x 3,0 m no centro de cada parcela foi delimitada com vistas à análise da produtividade da cultura.

O delineamento experimental foi em blocos completos ao acaso, constituído de cinco tratamentos e cinco repetições. Os tratamentos foram assim constituídos: $\mathrm{T}_{0}$ : Testemunha; $\mathrm{T}_{1}: 40$ t ha ${ }^{-1}$ de M.O.; $\mathrm{T}_{2}: 20 \mathrm{t} \mathrm{ha}^{-1}$ de gesso; $\mathrm{T}_{3}: 20 \mathrm{tha}^{-1}$ de gesso +40 tha ${ }^{-1}$ de M.O.; $\mathrm{T}_{4}$ : $10 \mathrm{t} \mathrm{ha}^{-1}$ de gesso $+20 \mathrm{t} \mathrm{ha}^{-1}$ de M.O. Aárea experimental foi de $105 \times 105 \mathrm{~m}$ e a parcela experimental de cada tratamento de $21 \times 21 \mathrm{~m}$.

A distribuição da matéria orgânica e do gesso agrícola foi realizada de forma manual, tendo-se o cuidado de proceder a uma uniformização de sua respectiva quantidade por tratamento; após a distribuição dos melhoradores químicos realizou-se a incorporação, através de uma gradagem (Figura 2).

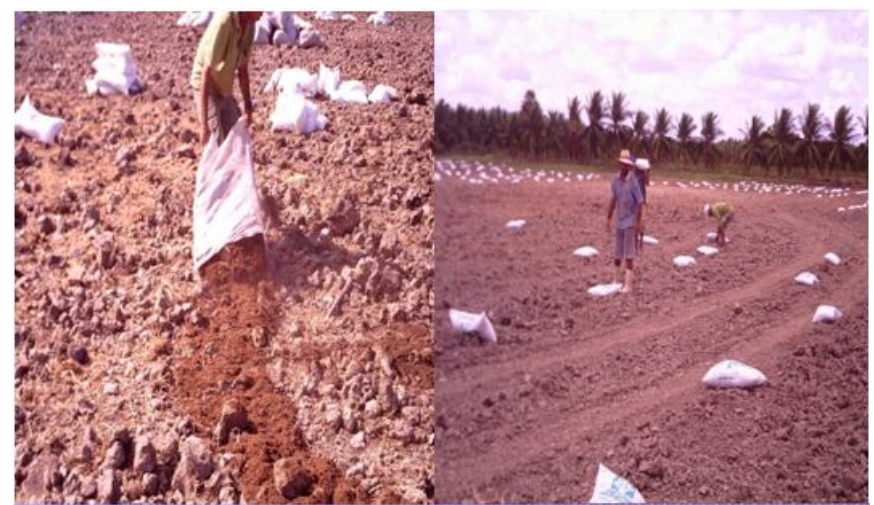

Figura 2. Distribuição de matéria orgânica e de gesso agrícola
A análise dos indicadores de rentabilidade foi realizada de forma "ex-ante" e "ex-post". Consideraram-se os custos e/ou investimentos na recuperação do solo sódico, além dos custos associados à instalação futura de um sistema de drenagem subterrânea. Os benefícios foram constituídos pelos valores brutos da produção, associados às culturas do feijoeiro, cultivar Epace-11, até o terceiro ano, e da cultura do coqueiro a partir do terceiro ano (Figura 3).

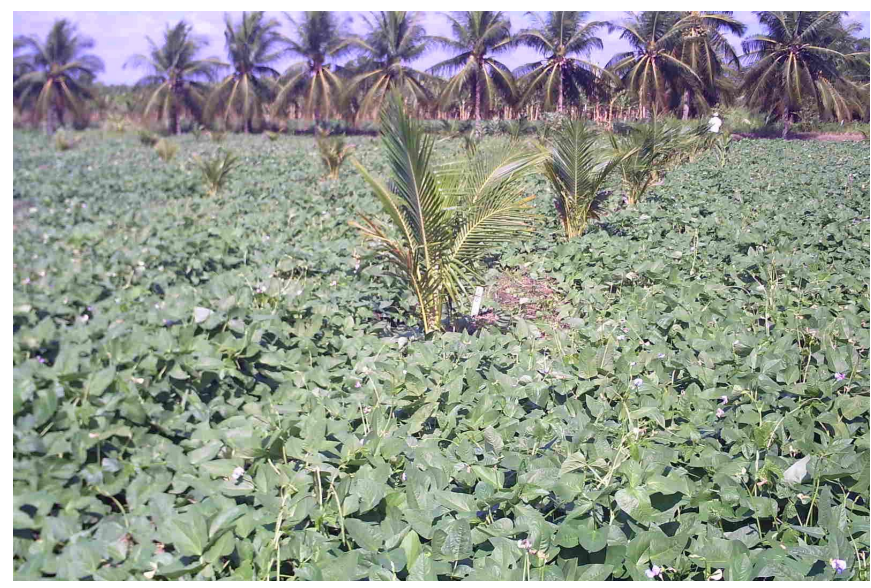

Figura 3. Cultivo de coqueiro consorciado com feijoeiro

Os dados de produtividade do feijoeiro foram medidos (análise ex-ante); já os dados de produtividade do coqueiro foram estimados (análise ex-post) com base nos dados de produtividade do coqueiro no Perímetro Irrigado Curu Pentecoste. A colheita ocorre em média a cada 35 dias, sendo que se consideraram as produtividades de 6, 9, 12 e 15 cocos planta $^{-1}$ colheita $^{-1}$ para os terceiro, quarto, quinto e sexto anos, respectivamente, quando sua produtividade se estabilizará.

A amortização dos investimentos da recuperação do solo sódico e a instalação do sistema de drenagem subterrânea foram realizadas em três anos, com período de carência de dois anos. Na Tabela 2 são apresentados os elementos econômicos para cálculo dos indicadores de rentabilidade, considerando-se um horizonte de 10 anos.

As receitas dos dois primeiros anos foram compostas pelo valor bruto da produção (VBP) da cultura do feijão-de-corda; no terceiro ano, pelo VBP das culturas do feijão-de-corda e do coqueiro, e nos demais anos, pelo VBP da cultura do coqueiro. Quanto aos custos do processo de recuperação eles se compuseram no primeiro ano do custo de produção da cultura do feijão-de-corda e custo de instalação e produção da cultura do coqueiro.

No segundo ano compôs-se do custo de produção da cultura do feijoeiro e manutenção da cultura do coqueiro. No terceiro ano do custo de produção e manutenção das culturas do coqueiro e feijoeiro, além da primeira parcela da amortização dos investimentos; nos quarto e quinto anos, do custo de manutenção da cultura do coqueiro e respectivas amortizações e, do sexto ao décimo anos, do custo de manutenção da cultura do coqueiro.

A análise da viabilidade econômica da recuperação do solo da área do experimento foi realizada com base nos indicadores de rentabilidade da análise de investimento: relação benefício/ 
Tabela 2. Dados para o cálculo dos indicadores de rentabilidade

\begin{tabular}{|c|c|c|c|c|c|}
\hline \multirow{2}{*}{ Ano* } & \multicolumn{2}{|c|}{ Valores nominais - $\mathbf{R} \$$} & \multirow{2}{*}{$\mathrm{FD} * *(12 \%)$} & \multicolumn{2}{|c|}{ Valores atualizados - R\$ } \\
\hline & Custos & Receitas & & Custos & Receitas \\
\hline 1 & $2.745,00$ & $1.473,00$ & 0,8929 & $2.451,00$ & $1.315,00$ \\
\hline 2 & $1.745,00$ & $1.473,00$ & 0,8929 & $1.558,11$ & $1.315,24$ \\
\hline 3 & $5.472,00$ & $3.167,00$ & 0,7972 & $4.362,28$ & $2.524,73$ \\
\hline 4 & $4.527,00$ & $2.543,00$ & 0,7118 & $3.222,32$ & $1.810,11$ \\
\hline 5 & $4.527,00$ & $3.391,00$ & 0,6355 & $2.876,91$ & $2.154,98$ \\
\hline 6 & 800,00 & $4,239,00$ & 0,5674 & 453,92 & $2.405,21$ \\
\hline 7 & 800,00 & $4,239,00$ & 0,5066 & 405,28 & $2.147,48$ \\
\hline 8 & 800,00 & $4,239,00$ & 0,4523 & 361,84 & $1.917,30$ \\
\hline 9 & 800,00 & $4,239,00$ & 0,4039 & 323,12 & $1.712,13$ \\
\hline 10 & 800,00 & $4,239,00$ & 0,3606 & 288,48 & $1.528,58$ \\
\hline Soma & $23.016,00$ & $33.242,00$ & & $16.597,26$ & $8.988,76$ \\
\hline
\end{tabular}

* Definiu-se como ano 10 primeiro ano do horizonte de planejamento do projeto, para efeito de análise do investimento

** Fator de desconto

custo, valor presente líquido, taxa interna de retorno e período "payback". A taxa mínima de atratividade de retorno considerada no estudo foi de $12 \%$ devendo, portanto, o valor da TIR superar este valor para que o projeto apresente viabilidade econômica.

\section{RESULTADOS E DISCUSSÃO}

A Tabela 3 apresenta o demonstrativo do custo de recuperação de 1,0 ha do solo sódico para cada um dos tratamentos. Os valores demonstram que na composição do custo de recuperação o uso e os níveis dos melhoradores químicos são relevantes na composição final do referido custo.

Tabela 3. Custo de recuperação do solo $\left(R \$\right.$ ha $\left.^{-1}\right)$ em função dos tratamentos

\begin{tabular}{cccccc}
\hline Trat. $^{*}$ & Subsolagem & $\begin{array}{c}\text { Limpeza } \\
\text { coletor }\end{array}$ & Gesso & $\begin{array}{c}\text { Mat. } \\
\text { orgânica }\end{array}$ & $\begin{array}{c}\text { Custo da } \\
\text { recuperação }\end{array}$ \\
$\mathrm{T}_{0}$ & 180,00 & 300,00 & - & - & 480,00 \\
$\mathrm{~T}_{1}$ & 180,00 & 300,00 & - & $4.200,00$ & $4.680,00$ \\
$\mathrm{~T}_{2}$ & 180,00 & 300,00 & $2.300,00$ & - & $2.780,00$ \\
$\mathrm{~T}_{3}$ & 180,00 & 300,00 & $2.300,00$ & $4.200,00$ & $6.980,00$ \\
$\mathrm{~T}_{4}$ & 180,00 & 300,00 & $1.150,00$ & $2.100,00$ & $3.730,00$ \\
\hline
\end{tabular}

* Significado dos tratamentos: $T_{0}$ : testemunha; $T_{1}: 40$ tha ${ }^{-1}$ de matéria orgânica; $T_{2:} 20$ t ha ${ }^{-1}$ de gesso; $\mathrm{T}_{3}: 20$ tha $^{-1}$ (gesso) +40 tha- $^{-1}$ (M.0); $\mathrm{T}_{4}: 10$ tha $^{-1}$ (gesso) $+20 \mathrm{tha}^{-1}$ (M.0)

Os preços de aquisição por tonelada, considerando-se os custos de transporte até a propriedade agrícola foram, na época do experimento, da ordem de $\mathrm{R} \$ 115,00$ e $\mathrm{R} \$ 105,00$ para o gesso e a matéria orgânica, respectivamente.

A Tabela 4 apresenta um demonstrativo do valor bruto da produção (VBP) gerado pela cultura do feijoeiro no processo de recuperação do solo sódico para cada um dos tratamentos.

Tabela 4. Receita bruta ( $\left.R \$ h^{-1}\right)$ do feijão-de-corda em função dos tratamentos

\begin{tabular}{|c|c|c|c|}
\hline Tratamento & $\begin{array}{l}\text { Prod. Média } \\
\left(\mathrm{kg} \mathrm{ha}^{-1}\right)\end{array}$ & $\begin{array}{c}\text { P.V. } \\
\left(R \$ \text { kg }^{-1}\right)\end{array}$ & $\begin{array}{c}\text { VBP }^{*} \\
\left(R \$ h^{-1}\right)\end{array}$ \\
\hline $\mathrm{T}_{0}$ & 446,68 a & 1,5 & 670,00 \\
\hline $\mathrm{T}_{1}$ & $666,14 a$ & 1,5 & 999,20 \\
\hline $\mathrm{T}_{2}$ & $604,56 a$ & 1,5 & 906,80 \\
\hline $\mathrm{T}_{3}$ & $982,26 a$ & 1,5 & $1.473,40$ \\
\hline $\mathrm{T}_{4}$ & $679,94 a$ & 1,5 & $1.019,90$ \\
\hline
\end{tabular}

*P.V. - preço de venda, VBP - valor bruto de produção
Tal qual na composição do custo de recuperação, os valores de VBP apresentam uma estreita relação com a aplicação e os referidos níveis dos melhoradores, que foram incorporados ao solo.

Esses valores demonstram ser bem mais atrativa, para o agricultor, a aquisição do gesso, considerando-se seu preço de aquisição e os resultados de produtividade obtidos com a cultura do feijoeiro, para as condições deste trabalho.

A produtividade do feijoeiro com a aplicação da matéria orgânica e do gesso, foi superior em $120 \%$ à produtividade sem o uso desses melhoradores, e inferior em apenas $18 \%$ à produtividade da cultura em solos sem problemas de sais do Perímetro.

Na Figura 4 se visualiza o comportamento do valor bruto da produção (VBP) do feijoeiro, em função do custo de recuperação do solo sódico para cada um dos tratamentos.

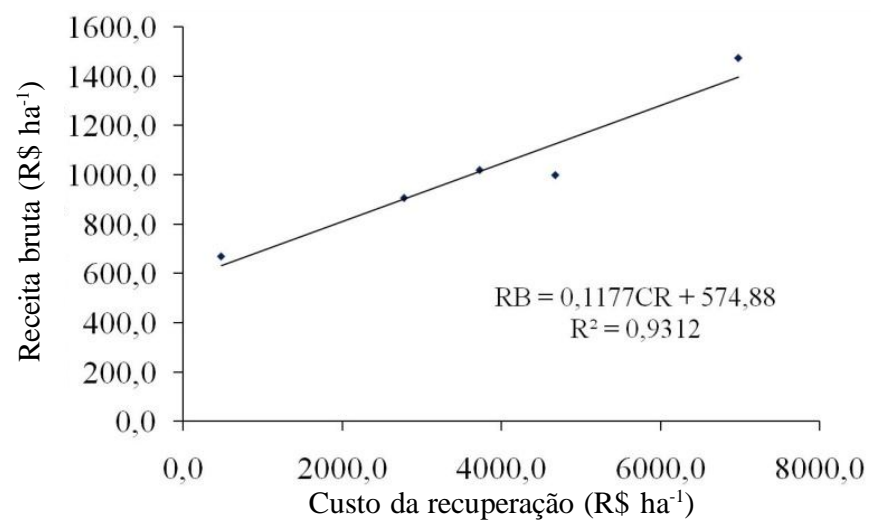

Figura 4. Valor bruto da produção em função do custo de recuperação

Verifica-se que o investimento no custo de recuperação proporcionou um retorno bruto de apenas $12 \%$ do valor alocado, comprovando a necessidade da geração de renda adicional com a introdução da cultura do coqueiro, a partir do terceiro ano.

Consideraram-se os custos e/ou investimentos e os benefícios associados ao tratamento $\mathrm{T}_{3}: 20 \mathrm{t} \mathrm{ha}^{-1}$ de gesso +40 $\mathrm{t} \mathrm{ha}^{-1}$ de matéria orgânica. Este tratamento apresentou o maior custo de recuperação e o maior valor bruto da produção, além 
de ter proporcionado a melhor condição de transmissão de água no perfil do solo.

Os indicadores de rentabilidade, considerando-se uma taxa de desconto de $12 \%$ ao ano, do processo de recuperação, foram de: relação benefício/custo (B/C) de 1,14; valor presente líquido (VPL) de R \$ 2.391,90 e taxa interna de retorno (TIR) igual a $20,86 \%$. Os referidos indicadores demonstram, com base nos critérios de decisão, viabilidade do processo de recuperação $(\mathrm{B} / \mathrm{C}>1,0$ e VPL $>0)$.

Quanto à taxa interna de retorno (20,86\%), principal indicador de rentabilidade de um projeto, por ser maior do que o custo de oportunidade do capital considerado na presente análise (12\%) mostra a viabilidade do referido processo.

Em outras palavras, a TIR igual a 20,86\% significa que o capital alocado no projeto suporta uma elevação da taxa de desconto de até 20,86 ao ano, para cada ano do horizonte de análise do projeto, ou seja, o referido investimento só será inviável se a taxa média de juros de mercado atingir valores superiores aos da referida taxa interna de retorno $(20,86 \%)$.

Valdivieso et al. (1988) obtiveram, em avaliação econômica da recuperação de solos salinos no Perímetro Irrigado de VazaBarris, Cocorobó, BA, valores de B/C iguais a 2,98 e TIR igual a $88,4 \%$. Costa et al. (2005) também verificaram, estudando indicadores de rentabilidade da recuperação de um solo sódico no Vale do Curu, Ceará, a viabilidade do processo de recuperação do solo.

Chinnapa \& Nagaraj (2007), numa avaliação econômica do impacto das intervenções públicas para melhoria da degradação dos solos afetados por sais através da tecnologia de drenagem subterrânea em Tungabhadra área do Projeto em Karnataka no sul da Índia, obtiveram valores de taxa de juros de $15 \%$ ao ano, a relação (B/C) de 1,54; o VPL de Rs (rúpias) 127.624 um "payback" de 0,58 ano. Os autores afirmam, ainda, que o governo deve ter, como objetivo, incentivar e educar os agricultores quanto à adoção da tecnologia de drenagem subterrânea, em grande escala.

Datta et al. (2000), verificaram um aumento na produtividade da cultura do trigo $200 \%$ superior, em Ismila, no período de 1986 a 1987. Em outras duas áreas, Ujhan e Bhana-Brahamana, observaram, nos primeiros quatro anos, um incremento de 30 a $40 \%$ na produtividade do trigo após a instalação do sistema de drenagem.

\section{ConclusõEs}

1. O cultivo do feijão-de-corda contribuiu com apenas $12 \%$ do processo de recuperação do solo sódico; do ponto de vista estritamente financeiro e na alternativa da aplicação de um único melhorador, o gesso se apresentou como a alternativa mais atrativa.

2. O processo de recuperação apresentou viabilidade econômica a uma taxa de juros de $12 \%$ ao ano, porém, considerando-se que o "payback" foi de nove anos, torna-se notória a necessidade da criação de uma linha de crédito subsidiada, destinada a agricultores familiares, como forma de incorporar, ao processo produtivo, as extensas áreas de solos degradados por sódio.

\section{AgradeCIMENTOS}

Os autores agradecem ao $\mathrm{CNPq}$, pelo financiamento da pesquisa.

\section{LITERATURA CITADA}

Amorim, J. R. de A.; Fernandes, P. D.; Gheyi, H. R.; Azevedo, N. C. de. Efeito da salinidade e modo de aplicação da água de irrigação no crescimento e produção de alho. Pesquisa Agropecuária Brasileira, v.37, p.167-176, 2002.

Batista, M. J.; Novaes, F.; Santos, D. G.; Suguino, H. H. Drenagem como instrumentos de dessalinização e prevenção da salinização de solos. Brasília: CODEVASF, 2002. 216p.

Chinnapa, B.; Nagaraj, N. An economic analysis of public interventions for amelioration of irrigation-induced soil degradation. Agricultural Economics Research Review, v.20, p.375-384, 2007.

Costa, R. N. T.; Saunders, L. C. U.; Oliveira Júnior, N. M. de; Biserra, J. V. Indicadores econômicos da recuperação de um solo sódico em condições de drenagem subterrânea no Vale do Curu, CE. Irriga, v.10, p.272-278, 2005.

Datta, K. K.; C. Dejong, Reclaiming salt-affected land through drainage in Haryana: A financial analysis, Agricultural Water Management, v.46, p.55-71, 2000.

Dutra, I.; Medeiros, J. F. de; Porto Filho, F. de Q.; Costa, M. da C. Determinação do fator de cobertura do melão cultivado sob diferentes lâminas e salinidades da água de irrigação. Revista Brasileira de Engenharia Agrícola e Ambiental, v.4, p.146-151, 2000.

Freire Filho, F. R.; Lima, J. A. A.; Ribeiro, V. Q. Feijão-caupi: Avanços tecnológicos. Brasília: Embrapa Informação Tecnológica, 2005. 519p.

Gheyi, H. R. Problemas de salinidade na agricultura irrigada. In: Oliveira, T. S.; Assis Jr., R. N.; Romero, R. E.; Silva, J. R. C. (ed.). Agricultura, sustentabilidade e o semiárido. Fortaleza: UFS/SBCS, 2000. p.329-346.

Horney, R. D.; Taylor, B.; Munk, D. S.; Roberts, B. A.; Lesch, S. M.; Plant, R. E. Development of practical site-specific management methods for reclaiming salt-affected soil. Computers and Electronics in Agriculture, v.46, p.379-397, 2005.

Keiffer, C. H.; Ungar, I. A. Germination and establishment of halophytes on brine-affected soils. Journal of Applied Ecology, v.39, p.402-415, 2002.

Leal, G. I.; Accioly, A de M.A.; Nascimento do A.W.C.; Freire, S dos G. B. M.; Montenegro, A de A.A.; Ferreira, L de F. Fitorremediação de solo salino por Atriplex nummularia e gesso de jazida. Revista Brasileira de Ciência do Solo, v.32, p.1065-1072, 2008.

Melo, M. R.; Barros, C. F de M.; Santos dos M. P.; Rolim, M. M. Correção de solos salino-sódicos pela aplicação de gesso mineral. Revista Brasileira de Engenharia Agrícola e Ambiental, v.12, p. 376-380, 2008. 
Sales, J. L.; Costa, R. N. T.; F. Filho, J. M.; Hernandez, F. F. F. Análise de desempenho de um sistema de drenagem subterrânea na cultura da videira no município de Jaguaruana, CE. Irriga, v.9, p.166-180, 2004.

Sartori, M. A. Análise de cenários de extração de óleo vegetal para a produção de biodisel na região de Minas Gerais. Viçosa: UFV, 2007. 88p. Dissertação Mestrado
Valdivieso, C. R.; Félix, S. G.; Cordeiro, G G Avaliação econômica da recuperação de solos salinos no perímetro de Vaza-BarrisCocorobó, BA. Petrolina: Centro de Pesquisa Agropecuária do Trópico Semi-Árido/EMBRAPA, 1988. 16p. Documentos, N.48 Viana, S. B. A.; Fernandes, P. D.; Gheyi, H. R. Germinação e formação de mudas de alface em diferentes níveis de salinidade de água. Revista Brasileira de Engenharia Agrícola e Ambiental, v.5, p.259-264, 2001. 